\title{
Qualitative Preference Modelling in Constraint Satisfaction
}

\author{
Yannis Dimopoulos ${ }^{1}$, Pavlos Moraitis ${ }^{2}$, and Alexis Tsoukiàs ${ }^{3}$ \\ 1 Computer Science Department, University of Cyprus \\ yannis@cs.ucy.ac.cy \\ 2 Dept. of Mathematics and Computer Science \\ University René Descartes-Paris 5 \\ pavlos@math-info.univ-paris5.fr \\ 3 LAMSADE - CNRS, Université Paris Dauphine \\ tsoukias@lamsade.dauphine.fr
}

\begin{abstract}
The paper addresses the problem of finding an appropriate formalism for the representation of preferences expressed on an ndimensional space of attributes and on different layers: generic, contextual and structural preferences.

The paper first introduces a general framework for preference modelling and then specialises it for the multi-layer case. It then shows that in the case we privilege computational efficiency an appropriate formalism can be the CP-nets one. More precisely we show how contextual and structural preferences can be seen as different types of constraint satisfaction problems to which apply some Ceteris-Paribus preferential reasoning.
\end{abstract}

\section{Introduction}

Preferences represent the basic notion for any decision support activity. One of the principal tasks within a decision aiding process is to model preferences in such a way that it is possible to derive a final recommendation for the decision maker (the preferences of whom have been modelled; see [23]).

The problem is that quite often the decision maker adopts preference statements in "natural language" which do not necessarily have a straightforward modelling. It is therefore necessary to use appropriate languages for preference modelling taking into account the specific context where the constructed model is going to be used (for more details see [10] and [20]). In this paper we focus our attention on situations where:

- preferences can be expressed on different layers representing different types of possible use as well as different moments of a decision process;

- it is necessary to be able to formulate a final recommendation for the decision maker efficiently (as in automatic decision devices) irrespectively of the dimension of the space of possible outcomes to compare (thus, computational efficiency is more important than expressiveness of the language).

In the paper we present an initial study of how problems of these type can be handled, establishing a three layer preference model (generic, contextual and 
structured preferences) and adopting the CP-nets formalism in order to compute efficiently a "best choice". The paper is organised as follows. In section 2 we present the notation used and the problem statement. In section 3 we introduce our three layer model. Section 4 shows how CP-nets can be used in order to model structured preferences in a constraint satisfaction problem, while section 5 extends dynamic constraint satisfaction problems in order to work with contextual preferences introducing preferences among active variables. Several examples illustrate the paper's definitions.

\section{Notation and Problem}

\subsection{Notation}

We use capital letters $X, Y, Z \cdots$, possibly subscribed, in order to represent discrete (infinite denumerable) domains where variables can range. We call such domains "attributes". We use capital letters $A, B, C \cdots$ to represent sets of objects which can be described by the set of attributes $D=\{X, Y, Z \cdots\}$. Elements of, say set $A$, are denoted by lowercase letters $a, b, c \cdots$. Each such element can be seen as a vector in the space $X \times Y \times Z \times \cdots$.

Besides the usual notation, we use capital letters $P, Q, R, I \cdots$, possibly subscribed, in order to represent binary relations applied to a set $A$ (therefore: $P, Q, R \cdots \subseteq A \times A)$. We use such relations as a formalism for understanding and studying statements of the type "a is preferred to b", "a is at least as good as b" etc. We use the conventions introduced in [20,21]. See also [15]. More precisely, given a binary relation $\succeq$ (to be read as "at least as good as"), we write: - $P(a, b)$ or $a \succ b$ iff $x \succeq y$ and $\neg(y \succeq x)$;

- $I(a, b)$ or $a \sim b$ iff $x \succeq y$ and $y \succeq x$;

- $J(a, b)$ or $a \asymp b$ iff $\neg(x \succeq y)$ and $\neg(y \succeq x)$;

and we call $P$ as "strict preference", $I$ as "indifference" and $J$ as "incomparability".

Definition 1. A preference structure is a collection $\langle P, Q, R \cdots\rangle$ of preference relations able to partition any universe of discourse $A$

- $P \cup Q \cup R \cup \cdots=A \times A$;

- $P \cap Q=P \cap R=Q \cap R=\cdots=\emptyset$.

It is easy to see that the relations $P, I, J$ previously defined establish a preference structure. Binary relations are characterised by the properties they satisfy. It is easy to show that by definition 1 the preference structure $\langle P, J, I\rangle$ satisfies the following properties:

- $P$ is irreflexive and asymmetric;

- $J$ is irreflexive and symmetric;

$-I$ is reflexive and symmetric.

A binary relation (used as characteristic relation of a preference structure) can satisfy several properties. We are concerned with ordering properties, that is different types of transitivity (transitivity, semi-transitivity, Ferrers-transitivity, 
etc.) and completeness. The satisfaction of certain properties uniquely characterises a "preference model". For instance we have:

- $\succeq$ is a weak order iff it is reflexive, strongly complete and transitive;

- $\succeq$ is a semi order iff it is complete, semi and Ferrers transitive;

$-\succeq$ is a partial order iff it is reflexive and transitive;

Definition 2. For a given set A, we call a criterion the application of a preference model to the set $A$.

If a criterion is derived from an attribute we may use the same notation for both of them. However, the reader should note that these are not equivalent concepts. Objects which are different in a certain attribute may not be necessarily preferred to each other. For instance, two objects whose price is $€ 10 \mathrm{~K}$ and $€ 10.1 \mathrm{~K}$ are different on the attribute price, but not necessarily there is a preference between them; a decision maker may be indifferent among objects if the difference in their price is less than 0.5 Euro. Moreover, the concept of preference is subjective. Different decision makers have different preferences comparing the same objects.

Occasionally preference models (which are binary relations) can be represented through functions which both represent them and preserve them (for instance, for a weak order we have: $x \succeq y$ iff $\exists f: A \mapsto \mathbb{R}$ such that $f(x) \geq f(y))$. Only certain types of preference models admit such numerical representation (for instance, they have to be complete, see more in [20]).

\subsection{Our problem}

1. We consider a set of attributes $\mathcal{X}=\left\{X_{1}, X_{2} \cdots X_{n}\right\}$. Each attribute is endowed with its domain $\left(\operatorname{dom}\left(X_{j}\right)\right)$. We consider a set of outcomes $O \subseteq$ $\operatorname{dom}\left(X_{1}\right) \times \operatorname{dom}\left(X_{2}\right) \times \cdots \operatorname{dom}\left(X_{n}\right)$ and unless there is a problem of confusion we adopt the notation $O \subseteq X_{1} \times X_{2} \times \cdots X_{n}$. We denote the $i$ th element of $\operatorname{dom}\left(X_{j}\right)$ by $x_{i j}$.

2. We consider an agent (a decision maker) able to make preference statements of different nature. For example:

- "I prefer $a$ to $b$ on criterion $X_{j}$ ";

- "I prefer $a$ to $b$ considering a set of attributes $\mathcal{X}^{*} \subseteq \mathcal{X}^{\prime}$;

- "If two objects are identical in all attributes (of a set $\mathcal{X}^{*}$ ) but one $\left(X_{j}\right)$, then I prefer objects with value $x_{j 1}$ to objects with value $x_{j 2}$ to objects with value $x_{j 3} \cdots$;

- "Attribute $X_{j}$ is more important than attribute $X_{i}$ " - ....

3. We are looking for a subset of $O$ (possibly of one element), say $\hat{O}$, such that $\hat{O}$ is the "best" choice when we consider the decision makers' preference statements all together. In reality this is a reductive problem statement with respect to the richness of real problem situations, but for the moment we are going to concentrate our efforts on this one. For further discussion on this issue see $[5,23]$. 
The problem of course is not new. It has been extensively studied in decision theory and more precisely in multiple criteria decision aiding methodology as well in decision under risk and uncertainty, see $[5,13,16,17,22,24,25]$. However, most of the above literature underestimates a number of issues which in other settings can be very important.

- The dimension of the space $X_{1} \times X_{2} \times \cdots X_{n}$. For a large number of attributes we can easily end up with a set of potential objects which is exponentially large. An explicit representation of the binary relation representing a preference in a highly dimensional space can be impossible. This problem can be partially surmounted through the use of functions that represent and preserve the binary relation, but such functions do not always exist and do not always have an intuitive meaning.

- The expressivity of the language in which preferences are handled can be a key issue (considering the construction of user friendly interfaces). Binary relations and their functional representation (if they exist) are very powerful, but not always fit the users requirements for communication.

- The necessity to be able to provide the "best choice" any time and under any circumstances (as often happens in automatic decision making settings). Decision theory is geared on the hypothesis that there is always a client somewhere to ask and obtain more information when it is difficult to make a decision, but this is not always the case.

- The possibility that during a decision process the information concerning some attributes as well as some preference statements may change, therefore requiring a revision of the "best choice" identified and of the agent's behaviour towards obtaining it.

In this paper we put forward some ideas for a general framework enabling to take into account "natural language" preference statements and use them in different settings (from aiding a client in a decision process to implementing an automatic decision making device). To achieve our purpose we employ findings from both decision theory and artificial intelligence (see $[1,2,8-12,26,27])$.

\section{Attributes, Preferences, Layers and Models}

In this section we establish some basic concepts which are going to be used in the rest of the paper.

1. When we establish a decision model, we may have attributes for defining a set of alternatives and attributes used to make a decision, and these two types of alternatives do not necessarily coincide. We call "definitional" the attributes used in order to establish the set of alternatives $A$ (and we denote them with $\mathcal{X}_{D}$ ) and "decisional" the attributes used in order to identify the best choice in $A$ (and we denote them with $\mathcal{X}_{\Delta}$ ). The reader should note that the set $A$ can be a subset of the cartesian product of the elements of $\mathcal{X}_{D}$ if we introduce a set of hard constraints. 
Example 1. Consider yourself in a restaurant. Your options consist of choosing a meal (meat, fish, vegetarian) and a drink (red wine, white wine, water). The set of alternatives coincide with the two attributes space, $A=\mathcal{X}=$ $X_{m} \times X_{d}$ (there are 9 alternatives). However, if you have to make a choice, you may consider also the attribute "cost", but such an attribute does not introduce any new alternative option. Moreover, you may be totally indifferent on what you drink, the choice being determined by reasoning about the cost and the meal.

2. Preferences can be expressed on different layers. We distinguish three such layers

- Generic preferences. These are expressed on one or more decisional attributes, but not on definitional ones. Decisional variables, such as time and cost, are "generic" in the sense that they are meaningful in many different decision making settings. They usually represent a "decision rule of thumb" allowing to reach a "best choice" when a thorough analysis of the available options is not possible or is useless. Consider example 1: if your have little money, you may just focus on the least expensive option, regardless of what you eat or you drink.

- Contextual preferences. These are expressed on a subset of the definitional attributes irrespectively of the rest. The subset of the variables on which these preferences are defined depends on the context, ie. the values assigned to other variables, hence the term "contextual". Other decisional attributes may be considered, but not necessarily. Such preferences are used when a partial analysis of the definitional attributes space is considered sufficient at least in order to start taking some decisions. To some extend contextual preferences are equivalent to a statement of "nil" importance as far as some definitional attributes are concerned. Contextual preferences result in comparing subsets of the set $A$ as wholes. Consider once more example 1: a contextual preference could be: "I prefer fish to vegetarian and this to meat". This will result in three equivalence classes (solutions including fish, the ones including vegetarian and the ones including meat) which are totally ordered: a weak order on $A$.

- Structural preferences. These are expressed using the whole set of definitional attributes. Other decision attributes may be considered, but not necessarily. Structural preferences imply using the whole definitional attributes space (possibly reduced by the hard constraints) and thus implies an extensive comparison of them. Consider once more example 1: although I may keep stating that I prefer fish to vegetarian to meat, I may add that I prefer red wine to white wine to water, but that I also prefer white wine with fish to red wine with meat etc.

In order to have a better understanding of these three different layers of preferences we present a more detailed example.

Example 2. Consider the situation where an agent has to decide what to do this evening. He may stay at home (reading a book or watching TV) or he may go 
out (to a movie, to a restaurant, to a bar). In the second case he may see a friend (Ann or Bob) or not. We have the following definitional attributes:

- type of activity, $X_{1}=\{$ inside,outside $\}$,

- inside activity, $X_{2}=\{$ Book, TV $\}$,

- outside activity, $X_{3}=\{$ Movie,Restaurant,Bar $\}$,

- friends, $X_{4}=\{$ Ann,Bob $\}$.

However, note that the assignment of the value "inside" to variable $X_{1}$ renders variable $X_{3}$ irrelevant. Indeed, if the agent decides to stay at home, she is not concerned with choosing a movie, a restaurant or a bar. Similarly, variable $X_{4}$ is also irrelevant in this case. If the definitional attributes $X_{3}$ and $X_{4}$ are irrelevant in this case, so are the possible preferences that the agent may have on these attributes. This is one of the cases where contextual preferences arise. Indeed, the values assigned to some of the variables, determine the definitional attributes and therefore the preferences that are defined in terms of these attributes.

The above problems belongs to the class of dynamic constraint satisfaction problems. In section 5 we discuss how CP-networks can be extended from classical to dynamic constraint satisfaction problems. To correctly represent our problem as a classical constraint satisfaction one, we introduce a new value in the domains of the variables, called null value and denoted by $\emptyset$. A variable assumes this value iff it is irrelevant given the values of the other variables. Therefore, the problem now becomes:

- type of activity, $X_{1}=\{$ inside,outside $\}$,

- inside activity, $X_{2}=\{$ Book, TV, $\emptyset\}$,

- outside activity, $X_{3}=\{$ Movie,Restaurant,Bar, $\emptyset\}$,

- friends, $X_{4}=\{$ Ann,Bob, $\emptyset\}$.

Typical alternatives are $\langle$ Outside,, , Restaurant,Bob $\rangle$ or $\langle$ Inside,TV,,,$\emptyset\rangle$. Potentially there are 72 such alternatives, but using hard constraints of the type "if $X_{1}=$ Inside, then $X_{2} \neq \emptyset$ and $X_{3}=X_{4}=\emptyset$ we can reduce its size down to 20 .

The four dimensions could be also criteria, provided the agent is able to express some preferences (at least a partial order). However, we may use two more criteria which are not among the above introduced definitional attributes: "time to implement a decision" and "cost of a decision". These are decisional attributes. Consider the attribute "time". We may have time values for each of the above attribute values allowing an additive computation of the time necessary to implement a decision. Consider for instance the alternatives introduced previously. Suppose we know that $t\left(x_{11}\right)=1, t\left(x_{12}\right)=10, t\left(x_{21}\right)=$ $5, t\left(x_{22}\right)=1, t\left(x_{23}\right)=10$. A solution $a=\langle$ Inside, $\mathrm{TV}, \emptyset, \emptyset\}$ will give $t(a)=$ $t\left(x_{11}\right)+t\left(x_{22}\right)+t\left(x_{34}\right)+t\left(x_{43}\right)=2$.

- Generic preferences do not take into account the definitional attributes and focus on one of the decisional attributes, say time. The preference model here is a simple weak order such that " $a$ is better than $b$ iff $t(a)<t(b)$ ". Applying this single criterion to the whole set $A$ will result in a weak order where a maximal element always exist (perhaps not necessarily with only 
one element. Clearly, this is a rapid way to identify a solution, possibly not very satisfactory.

- Contextual preferences are expressed on one or more definitional attributes through statements of the type: "I prefer to go out instead of staying at home" $\left(x_{21}>x_{11}\right)$ regardless of whatever else I am going to do and with whom" or "If I go out I prefer a movie to a restaurant to a bar, provided that I have the same company". The first statement results in a total order of two equivalence classes, the first of the type $\left\langle x_{12}, \emptyset, x_{3}, x_{4}\right\rangle$ and the second of the type $\left\langle x_{11}, \emptyset, x_{3}, x_{4}\right\rangle$ (equivalent to the result of a lexicographic order where $X_{1}$ is the most important criterion; see [14]). The second statement results in a partial order where only compare alternatives of the type $\left\langle x_{12}, \emptyset, x_{3 j}, x_{4 k}\right\rangle$ to alternatives of the type $\left\langle x_{12}, \emptyset, x_{3 i}, x_{4 k}\right\rangle$. A comparison to an alternative of the type $\left\langle x_{12}, \emptyset, x_{3 i}, x_{4 l}\right\rangle$ is impossible.

- Structural preferences are defined on the whole set of definitional attributes plus, possibly, other decisional ones. In this case we also allow any type of comparison among alternatives including conflicting statements and their relevant preference relations.

\section{Ceteris Paribus as structural preference}

In order to be able to provide a final recommendation to a decision maker, we have to solve a preference aggregation problem. With this term we refer to the problem of establishing an overall preference relation (an order on the set of outcomes) taking into account all the criteria the decision maker considers relevant to his problem. Unfortunately there is no universal way to solve this problem (see [4] and [5]). Basically, what we know is that, under looser conditions on the type of preferences to aggregate and properties to satisfy by the final result, the resulting preference relation is not an order (neither completeness nor acyclicity can be guaranteed: see [3]). If the stake is to obtain rapidly a reasonable recommendation, we have to simplify both the possible types of preference statements that can be modelled and aggregated, and the aggregation procedure itself. For this purpose, in this paper we have chosen the use of the CP-nets formalism which guarantees an efficient computation of a final result, although it is less expressive than other frameworks.

The reader should note that Ceteris-Paribus comparisons of multi-attribute outcomes is one of the simplest models used in order to perform such a comparison. In the literature (see [13], [17], [25]) there exist different models under which such a comparison is possible based on different assumptions about the dependencies among subsets of criteria. For a general theoretical framework the reader may refer to the literature on conjoint measurement (see [18] and the more recently $[6]$ ).

In this section we focus on the use of CP-nets as the basic formalism. We review the basic CP-network semantics in the spirit of [27].

Assume a constraint satisfaction problem $\mathcal{C}$ over a set of variables $\mathcal{X}=$ $\left\{X_{1}, \ldots, X_{n}\right\}$ with domains $\operatorname{dom}\left(X_{1}\right), \ldots, \operatorname{dom}\left(X_{n}\right)$ respectively. The set of possible outcomes of $\mathcal{C}$, is a subset of $\operatorname{dom}\left(X_{1}\right) \times \ldots \times \operatorname{dom}\left(X_{n}\right)$, each element of 
which satisfies all the constraints of $\mathcal{C}$. In order to simplify our discussion, in the following we specify a constraint satisfaction problem by referring to its set of outcomes. We assume that variable domains are pairwise disjoint, ie. for every $X_{i}, X_{j} \in \mathcal{X}$ it holds that $\operatorname{dom}\left(X_{i}\right) \cap \operatorname{dom}\left(X_{j}\right)=\emptyset$. Given the value assignments $x^{*}$ and $x^{* *}$ to the set of variables $\mathcal{X}^{*}$ and $\mathcal{X}^{* *}$ respectively with $\mathcal{X}^{* *} \subseteq \mathcal{X}^{*} \subseteq \mathcal{X}$, we write $x^{*} \models x^{* *}$ to denote that the projection of $x^{*}$ to the variables of $\mathcal{X}^{* *}$ equals $x^{* *}$.

A preference statement is an expression of the form $x^{*}: x_{i k} \succ x_{j k}$ where $x^{*}$ is an assignment to a set of variables $\mathcal{X}^{*} \subseteq \mathcal{X}$ and $x_{i k}, x_{j k}$ are values of a variable $X_{k}$ such that $X_{k} \cap \mathcal{X}^{*}=\emptyset$. We then write $x_{i k} \succ_{x^{*}} x_{j k}$. Intuitively, such a preference statement means that, given the assignment $x^{*}$, the value $x_{i k}$ is preferred to the value $x_{j k}$. A CP-network is set of preference statements on the set of variables $\mathcal{X}$ that is used to rank an associated set of possible outcomes. We define the set of parent $P a\left(X_{k}\right)$ of a variable $X_{k}$ in a CP-net $N$ as $P a\left(X_{k}\right)=\left\{y \mid y \in \mathcal{X}\right.$ and $N$ contains a statement of the form $x^{*}: x_{i k} \succ x_{j k}$ where $x_{i k}, x_{j k} \in \operatorname{dom}\left(X_{k}\right)$ and $x^{*}$ contains some value for $\left.y\right\}$. We assume that in each preference statements of the form $x^{*}: x_{i k} \succ x_{j k}$ on the values of variable $X_{k}$, the assignment $x^{*}$ is a complete assignment to the set of variables $\operatorname{Pa}\left(X_{k}\right)$.

A CP-net $N$ induces a graph $G_{N}$ that contains a node for every variable of $N$ and an edge from the node associated with variable $X_{j}$ to the node of variable $X_{i}$ if $X_{j} \in P a\left(X_{i}\right)$. We say that $N$ is acyclic iff $G_{N}$ is acyclic. The notation $\operatorname{tr}(R)$ denotes the transitive closure of a binary relation $R$.

Definition 3. [27]. Let $s=x^{*}: x_{i k} \succ q_{j k}$ be a preference statement. The relation induced by $s$ on a set of outcomes $O$ is a binary relation $R_{s}=\{(a, b) \mid a, b \in O$ and $a=\mathbf{w} x_{i k}$ and $b=\mathbf{w} x_{j k}$ and $\left.\mathbf{w} \models x^{*}\right\}$. The relation induced by $a C P$-net $N=\left\{s_{1}, s_{2}, \ldots s_{n}\right\}$ is the relation $R_{N}=\operatorname{tr}\left(R_{s_{1}} \cup R_{s_{2}} \cup \ldots \cup R_{s_{n}}\right)$.

Therefore, the criteria aggregation method used in the CP-networks is the disjunctive aggregation.

If $N$ is an acyclic CP-net the relation $R_{N}$ is a strict partial order, ie. it is irreflexive, asymmetric and transitive. The next theorem proves the first two properties of $R_{N}$, as transitivity follows from its definition.

Theorem 1. Let $N$ be an acyclic CP-net. Then, the relation $R_{N}$ is irreflexive and asymmetric.

If $N$ is an acyclic CP-network we say that the outcome $o_{i}$ is strictly preferred to outcome $o_{j}$ wrt $N$, denoted by $o_{i} \succ_{N} o_{j}$, if $\left(o_{i}, o_{j}\right) \in R_{N}$. We drop $N$ from $\succ_{N}$ when the CP-network to which we refer is clear from the context. The following example illustrates the ranking relation imposed on the set outcomes by the CP-networks.

Example 3. Let $N$ be the CP-network defined on the variables $X, Y, Z$ as follows:

$$
\begin{aligned}
& s_{1}=: x_{1} \succ x_{2} \quad s_{2}=x_{1}: y_{1} \succ y_{2} \\
& s_{3}=x_{2}: y_{2} \succ y_{1} \quad s_{4}=y_{1}: z_{1} \succ z_{2} \\
& s_{5}=y_{2}: z_{2} \succ z_{1}
\end{aligned}
$$


The relation induced by each of the above statements are the following:

$R_{s_{1}}=\left\{\left(x_{1} y_{1} z_{1}, x_{2} y_{1} z_{1}\right),\left(x_{1} y_{1} z_{2}, x_{2} y_{1} z_{2}\right),\left(x_{1} y_{2} z_{1}, x_{2} y_{2} z_{1}\right),\left(x_{1} y_{2} z_{2}, x_{2} y_{2} z_{2}\right)\right\}$

$R_{s_{2}}=\left\{\left(x_{1} y_{1} z_{1}, x_{1} y_{2} z_{1}\right),\left(x_{1} y_{1} z_{2}, x_{1} y_{2} z_{2}\right)\right\}$

$R_{s_{3}}=\left\{\left(x_{2} y_{2} z_{1}, x_{2} y_{1} z_{1}\right),\left(x_{2} y_{2} z_{2}, x_{2} y_{1} z_{2}\right)\right\}$

$R_{s_{4}}=\left\{\left(x_{1} y_{1} z_{1}, x_{1} y_{1} z_{2}\right),\left(x_{2} y_{1} z_{1}, x_{2} y_{1} z_{2}\right)\right\}$

$R_{s_{5}}=\left\{\left(x_{1} y_{2} z_{2}, x_{1} y_{2} z_{1}\right),\left(x_{2} y_{2} z_{2}, x_{2} y_{2} z_{1}\right)\right\}$

The ranking induced by the relation $R_{N}=\cup_{i=1}^{5} R_{s_{i}}$ specifies that $x_{1} y_{1} z_{1} \succ$ $x_{1} y_{1} z_{2} \succ x_{1} y_{2} z_{2} \succ\left\{x_{1} y_{2} z_{1}, x_{2} y_{2} z_{2}\right\} \succ x_{2} y_{2} z_{1} \succ x_{2} y_{1} z_{1} \succ x_{2} y_{1} z_{1}$. Note that the outcomes $x_{1} y_{2} z_{1}$ and $x_{2} y_{2} z_{2}$ are incomparable.

TCP-networks [7] extend CP-networks with relative variable importance statements. A relative variable importance (or relative importance) statement is of the form $x^{*}: X \triangleright Y$ where $X, Y \subseteq \mathcal{X}$, and the sets $P a(X),\{X\}$, and $\{Y\}$ are pairwise disjoint. Intuitively, the meaning of such a sentence is that when $x^{*}$ is true, whatever the value on attribute $Y$, is we prefer better values on attribute $X$. A variable importance statement induces a binary relation on the set of possible outcomes.

Definition 4. Let $v=x^{*}: X_{k} \triangleright X_{l}$ be a variable importance statement of a TCP-net $N$. The relation induced by $v$ on a set of outcomes $O$ is a binary relation $R_{v}=\left\{(a, b) \mid a, b \in O, a=\mathbf{w} \mathbf{z} x_{i k} x_{a l}, o_{j}=\mathbf{w} \mathbf{z} x_{j k} x_{b l}, x_{i k} \succ_{\mathbf{z}} x_{j l}\right.$, and $\left.\mathbf{w z}=x^{*}\right\}$, where $x_{i k}, x_{j k} \in \operatorname{dom}\left(X_{k}\right)$ and $x_{a l}, x_{b l} \in \operatorname{dom}\left(X_{l}\right)$. The relation induced by a TCP-net $N$ that contains the preference statements $s_{1}, s_{2}, \ldots s_{n}$ and the variable importance statements $v_{1}, v_{2}, \ldots v_{m}$ is $R_{N}=\operatorname{tr}\left(R_{s_{1}} \cup R_{s_{2}} \cup\right.$ $\left.\ldots \cup R_{s_{n}} \cup R_{v_{1}} \cup R_{v_{2}} \cup \ldots \cup R_{v_{m}}\right)$.

We can extend the notion of the graph $G_{N}$ associated with a CP-nets to the graph associated with a TCP-net $N$, also denoted by $G_{N}$, by adding to the graph an edge from the node that corresponds to $X$ to the node that corresponds to $Y$ for every variable importance statement of the form $x^{*}: X \triangleright Y$. We can now extend theorem 1 and show that for a TCP-network $N$ with an acyclic graph $G_{N}$ the relation $R_{N}$ is a strict partial order.

Theorem 2. Let $N$ be an acyclic TCP-net. Then, the relation $R_{N}$ is irreflexive and asymmetric.

As in the case of acyclic CP-networks we say that the outcome $a$ is strictly preferred to outcome $b$ wrt to an acyclic TCP-net $N$, denoted by $a \succ_{N} b$, if $(a, b) \in R_{N}$. The next example illustrates the semantics of TCP-networks.

Example 4. Consider the CP-network of example 3 extended with the variable importance $v=X \triangleright Z$. The associated binary relation is $R_{v}=\left\{\left(x_{1} y_{1} z_{1}, x_{2} y_{1} z_{2}\right)\right.$, $\left.\left(x_{1} y_{1} z_{2}, x_{2} y_{1} z_{1}\right),\left(x_{1} y_{2} z_{1}, x_{2} y_{2} z_{2}\right),\left(x_{1} y_{2} z_{2}, x_{2} y_{2} z_{1}\right)\right\}$. The reader should note that the relation $\operatorname{tr}\left(R_{s_{1}} \cup R_{s_{2}} \cup R_{s_{3}} \cup R_{s_{4}} \cup R_{s_{5}} \cup R_{v}\right)$ is antisymmetric and that the new relation includes the pair $\left(x_{1} y_{2} z_{1}, x_{2} y_{2} z_{2}\right)$, ie. outcomes that were previously incomparable now become comparable. 


\section{Contextual preferences and Dynamic Constraint Satisfaction}

As noted earlier, contextual preferences arise naturally in the context of dynamic constraint satisfaction problems. Moreover, such problems appear in many application domains such as configuration, compositional modelling, planning etc. In this section we extend CP-networks from classical to dynamic constraint satisfaction problems.

Dynamic Constraint Satisfaction [19] is an extension of classical constraint satisfaction with activity constraints that select the variables that participate in a solution. In the following we slightly change the notation and use the original definition of the problem as it presented in [19]. Formally, a dynamic constraint satisfaction problem $\mathcal{D}$ is a tuple of the form $\left.\mathcal{D}=<\mathcal{X}, \mathcal{X}_{I}, D, C^{C}, C^{A}\right\rangle$, where $\mathcal{X}=\left\{X_{1}, X_{2}, \ldots, X_{n}\right\}$ is a set of variables and $D=\left\{D_{1}, D_{2}, \ldots, D_{n}\right\}$ the domains of the variables that determine the possible values $D_{i}=\left\{x_{1 i}, x_{2 i}, \ldots, x_{k i}\right\}$ that can be assumed by the variables. The set $\mathcal{X}_{I}$ is the set of initial variables, and must appear in every solution, whereas $C^{C}$ the set of compatibility constraints and $C^{A}$ the set of activity constraints.

A variable is called active if it must be part of a solution. We declare that variable $X$ is active with the proposition active $(X)$, and that $X$ is not active with the proposition $\neg$ active $(X)$. Propositions of the form $\operatorname{active}(X)$ and $\neg \operatorname{active}(X)$ are called activity literals. The proposition active $(X)$ is satisfied in an outcome $a$ if $a$ assigns a value to $X$ and $\neg$ active $(X)$ is satisfied in $a$ if the variable $X$ does not appear in $a$. An activity constraint is an expression of the form

$c \rightarrow l_{1} \vee l_{2} \vee \ldots \vee l_{n}$

where each $l_{i}$ is an activity literal and $c$ is the activity condition. In the following we assume that each positive activity literal (ie. literal of the form active $(X)$ ) appears positively or negatively (ie. active $(X)$ or $\neg$ active $(X)$ ) in only one activity constraint. An activity condition is a conjunction of the form $V \wedge A$ where $A$ is a conjunction of activity literals and $V$ is a conjunction of expressions of the form $v_{i}=u_{i j}$ where $v_{i} \in \mathcal{X}$ and $u_{i j} \in D_{i}$. An activity constraint $c \rightarrow l_{1} \vee l_{2} \vee \ldots \vee l_{n}$ is satisfied in an outcome $a$ if either $c$ is not satisfied in $O$ or $l_{1} \vee l_{2} \vee \ldots \vee l_{n}$ is satisfied in $a$.

Given a dynamic constraint satisfaction problem $\mathcal{D}$, we can construct the activity graph $G_{\mathcal{D}}$ of $\mathcal{D}$ from its set of activity constraints $C^{A}$ as follows. For each positive activity literal active $\left(X_{i}\right)$ that appears in $C^{A}$, a corresponding node $n_{i}$ is included in $G_{\mathcal{D}}$. If $\mathcal{D}$ contains an activity constraint of the form $c \rightarrow l_{1} \vee l_{2} \vee \ldots \vee l_{n}$ such that some activity literal active $\left(X_{i}\right)$ belongs to $c$ and $l_{k}=\operatorname{active}\left(X_{j}\right)$ for some $1 \leq k \leq n$, then $G_{\mathcal{D}}$ contains the edge $\left(n_{i}, n_{j}\right)$. In this paper we restrict our attention to problems with an acyclic activity graph. We denote by $\operatorname{pred}_{\mathcal{D}}\left(X_{i}\right)$ the set $\operatorname{pred}_{\mathcal{D}}\left(X_{i}\right)=\left\{X_{j} \mid X_{j}\right.$ is a variable such that $n_{i}$ is reachable from $n_{j}$ in $G_{\mathcal{D}}$.

A compatibility constraint is a traditional constraint. A compatibility constraint $c$ is satisfied in an outcome if either some of the variables of $c$ are not active in $a$ or $c$ is satisfied in the traditional sense. 
A value assignment (or valuation) $a$ to a set of variables $\mathcal{X}^{\prime}$ such that $\mathcal{X}_{I} \subseteq$ $\mathcal{X}^{\prime} \subseteq \mathcal{X}$ of a dynamic constraint satisfaction problem $\mathcal{D}$ is called an outcome of $\mathcal{D}$ if $a$ satisfies all constraints of $C^{A} \cup C^{C}$ and no subset of $a$ is an outcome of $\mathcal{D}$.

We extend classical dynamic constraint satisfaction by introducing the concept of activity preference. An activity preference is a statement of the form

$$
c: \operatorname{active}\left(X_{i}\right) \succ \operatorname{active}\left(X_{j}\right)
$$

with the intuitive meaning that if the activity condition $c$ is satisfied we prefer that $X_{i}$ being active over $X_{j}$ being active. We assume that for every activity preference of the form $c: \operatorname{active}\left(X_{i}\right) \succ \operatorname{active}\left(X_{j}\right)$ that is contained in a dynamic constraint satisfaction problem $\mathcal{D}, \mathcal{D}$ also contains an activity constraint of the form $c \rightarrow$ active $\left(X_{1}\right) \vee$ active $\left(X_{2}\right) \vee \ldots \vee$ active $\left(X_{k}\right)$, with $\left\{X_{i}, X_{j}\right\} \subseteq\left\{X_{1}, X_{2}, \ldots, X_{k}\right\}$. Formally, the semantics of an activity preference statement is as follows.

Definition 5. Let $s=c$ : active $\left(X_{i}\right) \succ$ active $\left(X_{j}\right)$ be an activity preference statement. The ranking of the set of outcomes $O$ wrt to $s$ is a binary relation $R_{s}=\left\{(a, b) \mid a, b \in O\right.$ and $a=\mathbf{w} x_{m i}, b=\mathbf{w} x_{k j}$ where $x_{m i} \in D_{i}, x_{k j} \in D_{j}$, $\mathbf{w}=c$, and $\mathbf{w}$ is a value assignment to a subset of $\mathcal{X}$ that does not contain $X_{i}$ and $X_{j}$.

In addition to the above, we also slightly extend the language of CP-network in order to accommodate the activity literals. More specifically we allow preference statements of the form $s=p_{1} \wedge p_{2} \wedge \ldots \wedge p_{i} \wedge a_{1} \wedge a_{2} \wedge \ldots \wedge a_{j}: u_{1} \succ u_{2}$ where each $p_{m} \in D_{m}$ with $1 \leq m \leq i$, the $a_{i}$ 's are activity literals, and $\left\{u_{1}, u_{2}\right\} \subseteq D_{v}$ for some variable $X$. To distinguish between activity preference statements and preference statements on the values of variables, we call the later object level preference statements. We say that the object level preference statement $s$ is active if all the variables in the set $\left\{X, X_{1}, X_{2}, \ldots, X_{i}\right\}$ are active. Otherwise $s$ is inactive. The semantics of such a preference statement is defined as follows.

Definition 6. Let $s=p_{1} \wedge p_{2} \wedge \ldots \wedge p_{i} \wedge a_{1} \wedge a_{2} \wedge \ldots \wedge a_{j}: u_{1} \succ u_{2}$ be an object level preference statement of a dynamic constraint satisfaction problem with preferences $\mathcal{D}$ with $\left\{u_{1}, u_{2}\right\} \in D_{X}$. The ranking of the set of outcomes $O$ wrt to $s$ is a binary relation $R_{s}=\left\{(a, b) \mid a, b \in O\right.$ where $a=p_{1} p_{2} \ldots p_{n} \mathbf{z w}_{\mathbf{1}} u_{1}$, $b=p_{1} p_{2} \ldots p_{n} \mathbf{z w}_{\mathbf{2}} u_{2}$ and $\mathbf{z}$ is a value assignment to the variables of $\operatorname{pred}_{P}(v)$ and $\mathbf{w}_{\mathbf{1}}$ and $\mathbf{w}_{\mathbf{2}}$ are value assignments to a set of variables that do not belong to $\left.\operatorname{pred}_{\mathcal{D}}(X)\right\}$.

The semantics of variable importance statements, which are also extended to accommodate activity literals, is changed analogously. The new type of constraint satisfaction problems is defined formally as follows.

Definition 7. A dynamic constraint satisfaction problem with preferences $\mathcal{D}$, is a tuple of the form $\mathcal{D}=<\mathcal{X}, \mathcal{X}_{I}, D, C^{C}, C^{A}, A, S, F>$, where $\mathcal{D}^{\prime}=<\mathcal{X}, \mathcal{X}_{I}, D$, $C^{C}, C^{A}>$ is a classical dynamic constraint satisfaction problem and $A$ is a set 
of activity preferences, $S$ a set of variable importance statements and $F$ a set of object level preferences.

The ranking on the solutions induced by such a problem is defined as follows.

Definition 8. Let $\mathcal{D}=<\mathcal{X}, \mathcal{X}_{I}, D, C^{C}, C^{A}, A, S, F>$ be a dynamic constraint satisfaction problem with $A=\left\{a_{1}, a_{2}, \ldots, a_{k}\right\}, S=\left\{s_{1}, s_{2}, \ldots s_{n}\right\}$ and $F=$ $\left\{p_{1}, p_{2}, \ldots, p_{m}\right\}$. If $a, b$ are two outcomes of $\left.\mathcal{D}^{\prime}=<\mathcal{X}, \mathcal{X}_{I}, D, C^{C}, C^{A}\right\rangle$, it holds that $a \succ_{\mathcal{D}} b$ iff $(a, b) \in \operatorname{tr}\left(R_{a_{1}} \cup R_{a_{2}} \cup \ldots \cup R_{a_{k}} \cup R_{p_{1}} \cup R_{p_{2}} \cup \ldots \cup R_{p_{m}} \cup R_{s_{1}} \cup R_{s_{2}} \cup\right.$ $\left.\ldots \cup R_{s_{n}}\right)$.

The next example illustrates the new semantics.

Example 5. Consider the dynamic constraint satisfaction problem $P$ on the variables:

Where $=\{$ in, out $\}$

Book $=\{$ scifi, novel $\}$

$T V=\{$ action, comedy $\}$

Restaurant $=\{$ italian, indian $\}$

Bar $=\{$ winebar, discobar $\}$

Company $=\{A n n, B o b\}$

The activity preference statements are

$a_{1}=$ in $: \operatorname{active}(B o o k) \succ \operatorname{active}(T V)$

$a_{2}=$ out $:$ active (Restaurant) $\succ$ active $($ Bar $)$

There is also the variable importance statements

$s_{1}=\operatorname{active}($ Restaurant $):$ Company $\triangleright$ Restaurant

$s_{2}=\operatorname{active}($ Bar $):$ Bar $\triangleright$ Company

Finally the object level preferences are

$p_{1}=:$ out $\succ$ in $\quad p_{2}=:$ Ann $\succ$ Bob

$p_{3}=:$ novel $\succ$ scifi $\quad p_{4}=:$ action $\succ$ comedy

$p_{5}=:$ italian $\succ$ indian $\quad p_{6}=:$ discobar $\succ$ winebar

Finally, the activity constraints are

in $\rightarrow$ active $($ Book $) \vee$ active $(T V)$

out $\rightarrow \operatorname{active}($ Movie $) \vee$ active $($ Restaurant $) \vee \operatorname{active}($ Bar $)$

out $\rightarrow$ active (Company)

The set of outcomes of $P$ is $O=\{\{$ in, scifi $\},\{$ in, novel $\},\{$ in, action $\},\{$ in, comedy $\},\{$ out, italian, Ann $\},\{$ out, indian, Ann $\},\{$ out, italian, Bob $\},\{$ out, indian, Bob $\}$, $\{$ out, winebar, Ann $\},\{$ out, discobar, Ann $\},\{$ out, winebar, Bob $\},\{$ out, discobar, Bob $\}$.

The various preference statements induce the following binary relations on the set of outcomes $O$.

$R_{a_{1}}=\{(\{$ in, scifi $\},\{$ in, action $\}),(\{$ in, scifi $\},\{$ in, comed $\}),(\{$ in, novel $\},\{$ in, action $\})$, (\{in, novel $\},\{$ in , comedy $\})\}$.

$R_{a_{2}}=\{(\{$ out, italian, Bob $\},\{$ out, winebar, Bob $\}),(\{$ out, indian, Bob $\},\{$ out, winebar, Bob $\})$, (\{out, italian, Bob\}, \{out, discobar, Bob $\}),(\{$ out, indian, Bob $\},\{$ out, discobar, Bob $\})$,

(\{out, italian, Ann $\},\{$ out, winebar, Ann $\}),(\{$ out, indian, Ann $\},\{$ out, winebar, Ann $\})$, $(\{$ out, italian, Ann $\},\{$ out, discobar, Ann $\}),(\{$ out, indian, Ann $\},\{$ out, discobar, Ann $\})\}$ 
$R_{s_{1}}=\{(\{$ out, indian, Ann $\},\{$ out, italian, Bob $\})\}$

$R_{s_{2}}=\{(\{$ out, discobar, Bob $\},\{$ out, winebar, Ann $\})\}$

$R_{p_{1}}=\left\{\left(\left\{\right.\right.\right.$ out $\left., u_{1}, u_{2}\right\},\left\{\right.$ in,$\left.\left.u_{3}\right\},\right) \mid u_{1} \in D_{\text {Restaurant }} \cup D_{\text {Bar }}, u_{2} \in D_{\text {Company }}, u_{3} \in$

$\left.D_{\text {Book }} \cup D_{T V}\right\}$

$R_{p_{2}}=\{(\{$ out, discobar, Ann $\},\{$ out, discobar, Bob $\})(\{$ out, winebar, Ann $\},\{$ out, winebar, $B o b\})\}$

$R_{p_{3}}=\{(\{$ in, novel $\},\{$ in, scifi $\})\} \quad R_{p_{4}}=\{(\{$ in, action $\},\{$ in, comedy $\})\}$

$R_{p_{5}}=\{(\{$ out, italian, Ann $\},\{$ out, indian, Ann $\})(\{$ out, italian, Bob $\},\{$ out, indian, $B o b\})\}$

$R_{p_{6}}=\{(\{$ out, discobar, Ann $\},\{$ out, winebar, Ann $\})(\{$ out, discobar, Bob $\},\{$ out, winebar, $B o b\})\}$

The semantics of $P$ is given by the relation $R=\operatorname{tr}\left(R_{a_{1}} \cup R_{a_{2}} \cup R_{p_{1}} \cup R_{p_{2}}\right.$ $\cup \ldots \cup R_{p_{6}} \cup R_{s_{1}} \cup R_{s_{2}}$ ) which induced the following ranking on the set of outcomes $O$ :

\section{Conclusions}

In this paper we address a general preference modelling and aggregation problem. Suppose an n-dimensional attribute space where outcomes of potential actions can be compared. A possible "best choice" is searched. However, in our setting we introduce a further modelling component. We consider that preferences can be expressed on different layers:

- generic preferences: where attributes not used for describing the outcomes are used in order to make a decision; a decision rule of thumb allowing to obtain rapidly reasonable decisions, at least as far as some rough evaluations of the outcomes are concerned;

- contextual preferences: where only part of the attributes describing the outcomes are used to compare them, considering a specific context within which preferences have to be expressed;

- structural preferences: where the whole set of attributes is used in order to model the preferences of the decision maker.

At the same time we have chosen in our setting to privilege computational efficiency with respect to the expressiveness of the modelling language. For this purpose, as far as the modelling of contextual and structural preferences are concerned, we use the CP-nets formalism although limited in the allowed comparisons. Indeed structural preferences in the paper are modeled through conventional CP-net and TCP-nets.

The modelling of contextual preferences introduces the necessity to extend the language in order to take into account the dynamic nature of "contextual" preference statements. Indeed, in these cases we have to model statements where parts of the attribute space is simply not considered as it is irrelevant to the decision. The solution is to associate classic CP-nets to dynamic constraint satisfaction problems and introduce preferences as far as the activation of variables is concerned. 
This preliminary study opens a certain number of problems which we hope to handle in future research:

- a more thorough study of the computational complexity of the solution proposed in this paper;

- further extension of the CP-nets language in order to allow the comparison of more complex outcomes instead of Ceteris Paribus ones only;

- further analysis of the dynamics implicitly present in the idea of contextual preferences and the relations they have with the problem of revising preference statements and models.

\section{References}

1. C. Boutilier. Toward a logic for qualitative decision theory. In Proceedings of the 4th International Conference on Knowledge Representation and Reasoning, KR'94, pages 75-86. Morgan Kaufmann, San Francisco, 1994.

2. C. Boutilier, R. Brafman, H. Hoos, and D. Poole. Reasoning with conditional ceteris paribus preference statements. In Proceedings of the 15th Conference on Uncertainty in Artificial Intelligence, UAI'99, pages 71-80. Morgan Kaufmann, San Francisco, 1999.

3. D. Bouyssou. Outranking relations: do they have special properties? Journal of Multi-Criteria Decision Analysis, 5:99-111, 1996.

4. D. Bouyssou, T. Marchant, M. Pirlot, P. Perny, A. Tsoukiàs, and P. Vincke. Evaluation and decision models: a critical perspective. Kluwer Academic, Dordrecht, 2000.

5. D. Bouyssou, T. Marchant, M. Pirlot, P. Perny, A. Tsoukiàs, and P. Vincke. Evaluation and decision models: stepping stones for the analyst. Kluwer Academic, Dordrecht, forthcoming.

6. D. Bouyssou and M. Pirlot. Conjoint measurement tools for MCDM. In J. Figueira, S. Greco, and M. Ehrgott, editors, Multiple Criteria Decision Analysis: State of the Art Surveys, pages 73-132. Springer Verlag, Boston, Dordrecht, London, 2005.

7. R. Brafman and C. Domshlak. Introducing variable importance tradeoffs into CP-Nets. In Proceedings of UAI-2002, pages $69-76,2002$.

8. R. Brafman and M. Tennenholtz. On the foundations of qualitative decision theory. In Proceedings of the 13th National Conference on Artificial Intelligence, AAAI96, pages 1291-1296. MIT Press, Cambridge, 1996.

9. R. Brafman and M. Tennenholtz. Modeling agents as qualitative decision makers. Artificial Intelligence, 94:217-268, 1997.

10. J. Doyle. Prospects for preferences. Computational Intelligence, 20:111136, 2004.

11. J. Doyle and M. Wellman. Representing preferences as ceteris paribus comparatives. In Decision-Theoretic Planning: Papers from the 1994 Spring AAAI Symposium, pages 69-75. AAAI Press, Menlo Park, California, 1994.

12. D. Dubois, H. Fargier, P. Perny, and H. Prade. Qualitative decision theory: from Savage's axioms to non-monotonic reasoning. Journal of the ACM, 49:455-495, 2002.

13. P. Fishburn. Utility Theory for Decision Making. Wiley, New York, 1970.

14. P. Fishburn. Lexicographic orders, utilities and decision rules: a survey. Management Science, 20:1442-1471, 1974.

15. P. Fishburn. Preference structures and their numerical representations. Theoretical Computer Science, 217(2):359-383, April 1999. 
16. S. French. Decision theory - An introduction to the mathematics of rationality. Ellis Horwood, Chichester, 1988.

17. R. Keeney and H. Raiffa. Decisions with multiple objectives: Preferences and value tradeoffs. J. Wiley, New York, 1976.

18. D. Krantz, R. Luce, P. Suppes, and A. Tversky. Foundations of measurement, volume 1: Additive and polynomial representations. Academic Press, New York, 1971.

19. S. Mittal and B. Falkenhainer. Dynamic constraint satisfaction problems. In Proceedings of AAAI-1990, pages 25 - 32, 1990.

20. M. Öztürk, A. Tsoukiàs, and Ph. Vincke. Preference modelling. In M. Ehrgott, S. Greco, and J. Figueira, editors, State of the Art in Multiple Criteria Decision Analysis, pages 27 - 72. Springer Verlag, Berlin, 2005.

21. M. Roubens and P. Vincke. Preference Modeling. LNEMS 250, Springer Verlag, Berlin, 1985.

22. L. Savage. The Foundations of Statistics. J. Wiley, New York, 1954. second revised edition, 1972.

23. A. Tsoukiàs. On the concept of decision aiding process. Annals of Operations Research. To appear; appeared previously as DIMACS 2003-38 technical report, Rutgers University.

24. P. Vincke. Multicriteria Decision-Aid. J. Wiley, New York, 1992.

25. P. Wakker. Additive representations of preferences - A new foundation of decision analysis. Kluwer Academic, Dordrecht, 1989.

26. N. Wilson. Consistency and constrained optimisation for conditional preferences. In Proceedings of ECAI-04, pages 888 - 894, 2004.

27. N. Wilson. Extending CP-nets with stronger conditional preference statements. In Proceedings of AAAI-04, pages 735 - 741, 2004. 